\title{
Ewing's Sarcoma of the Humerus in a Three year-old boy Requiring Radical En-Bloc resection and Total Humeral Expandable Prosthetic Replacement: A Case Report
}

\section{Don Young Park*, Edwin Batista and Lawrence A Rinsky}

Department of Orthopedic Surgery, Stanford University Medical Center, Stanford California,USA

\begin{abstract}
Ewing's sarcoma is a malignant bone tumor that affects children typically in their second decade, with a low incidence in patients less than five years of age. Presenting symptoms may be nonspecific and may include pain, swelling, fever, and weight loss. Definitive diagnosis may be difficult and further investigation may be necessary to differentiate Ewing's sarcomas with osteomyelitis. We present a case report of a three year-old boy with Ewing's sarcoma that involved the entire right humerus. Due to his young age, and nonspecific nature of his presentation, the patient was initially misdiagnosed and treated for osteomyelitis. The patient required multi-agent chemotherapy and radical en-bloc resection of the entire right humerus, given the extent of his tumor involvement. A custom expandable modular humeral prosthesis was designed and engineered specifically for this patient. His arm is very functional three years postoperatively, and he is disease free. To our knowledge, this is the youngest report of Ewing's sarcoma that required total humeral resection and prosthetic replacement presented in the literature.
\end{abstract}

\section{Introduction}

Ewing's sarcoma, a small round blue cell tumor, is the second most prevalent primary malignant bone cancer in children, following osteosarcoma [1]. It belongs in the Ewing sarcoma family of tumors (ESFT), a group of tumors that also includes primitive neuroectodermal tumor (PNET) and Askin tumor [2]. Small round cell neoplasms and a common chromosomal translocation characterizes ESFT. Approximately 95\% of Ewing's sarcoma patients have a $\mathrm{t}(11 ; 22)$ or $\mathrm{t}(21 ; 22)$ translocation, resulting in $E W S$ and either FLI1 (chromosome 11) or ERG (chromosome 21) gene fusion [3]. In the United States, the incidence of Ewing's sarcoma is estimated at 2-3 cases per million children and has not changed over a 30 year time period $[4,5]$. Typically, Ewing's sarcoma has a peak incidence in the second decade but has been reported in all age groups [5]. In patients less than 5 years of age, the annual incidence is low, estimated at 0.6 per million [2]. In addition, only $3 \%$ of Ewing's sarcoma occurs in patients younger than 3 years of age [6]. Ewing's sarcoma can occur in the axial and appendicular skeleton with nearly equal frequency [3]. Tumors of the humerus occur infrequently with approximately $10 \%$ of Ewing's sarcoma found in this location [7]. Presenting symptoms may include pain, swelling, fever, and weight loss, which can be consistent in more common conditions, such as osteomyelitis, [1-3]. The classic radiographic appearance of Ewing's sarcoma With "onionskinning" periosteal reaction also can be seen in osteomyelitis [8]. Due to the Nonspecific nature of presentation, definitive diagnosis may be difficult and further investigation, including imaging studies and open biopsy may be necessary. We present a case of a three year-old boy with Ewing's sarcoma that involved the entire right humerus.

The patient required multi-agent chemotherapy and radical en-bloc resection of the Entire right humerus and replacement with a custom expandable modular humeral Prosthesis. To our knowledge, this is the youngest report of Ewing's sarcoma that Required total humeral resection and prosthetic replacement presented in the literature.

\section{Case Report}

A three-year-old previously healthy boy presented to our General Pediatric Service in April of 2008 with a two-week history of atraumatic right upper arm pain. He initially reported general malaise with fever and anorexia and began pointing to his right upper arm as the source of pain. The patient avoided using his right upper extremity.
Examination did not reveal a palpable mass or swelling and no skin lesions were seen. His initial inflammatory markers, including erythrocyte sedimentation rate and c-reactive protein, were found to be elevated. An MRI was obtained, which demonstrated abnormal bone marrow signal within the majority of the humeral shaft with irregular cortical borders, periosteal reaction, and mottling of the diaphysis with contrast administration (Figure 1).

These findings suggested osteomyelitis of the humerus, and the patient completed a sixweek course of antibiotics with complete resolution of his symptoms. His elevated inflammatory markers normalized after treatment. Radiographs performed one month after the onset of symptoms demonstrated cortical remodeling of the humeral diaphysis with minimal heterogeneity of the medullar space (Figure 2a,b). This finding, along with the patient's clinical improvement with antibiotic treatment, suggested appropriate treatment of the presumed osteomyelitis. In July of 2008 , the patient's symptoms recurred and a repeat $\mathrm{x}$-ray demonstrated heterogeneous mineralization of the right humerus, progression of periosteal reaction, with development of new ill-defined lucencies in the proximal humerus (Figure 3a,b). However, his inflammatory markers remained within normal limits. Given the recurrence and radiographic progression of the lesion, an open biopsy and culture was performed. Intraoperatively, abnormal appearing tissue was found within the medullary canal with no inflammatory purulent tissue. Intraoperative culture results proved to be negative. Pathology demonstrated malignant, small round blue cell tumor and cytogenetic analysis demonstrated ESW translocation by FISH analysis. Metastatic work-up including a bone scan found no detectable metastatic disease.

*Corresponding author: Don Young Park, MD, 300 Pasteur Dr. Stanford, CA 94305, USA, Tel: 650497 8201; Fax: 650-497-8891; E-mail: dypark@stanford.edu

Received September 13, 2011; Accepted November 17, 2011; Published November 18, 2011

Citation: Park DY, Batista E, Rinsky LA (2011) Ewing's Sarcoma of the Humerus in a Three year-old boy Requiring Radical En-Bloc resection and Total Humeral Expandable Prosthetic Replacement: A Case Report. Surgery 1:101. doi:10.4172/2161-1076.1000101

Copyright: () 2011 Park DY, et al. This is an open-access article distributed under the terms of the Creative Commons Attribution License, which permits unrestricted use, distribution, and reproduction in any medium, provided the original author and source are credited. 
Citation: Park DY, Batista E, Rinsky LA (2011) Ewing's Sarcoma of the Humerus in a Three year-old boy Requiring Radical En-Bloc resection and Total Humeral Expandable Prosthetic Replacement: A Case Report. Surgery 1:101. doi:10.4172/2161-1076.1000101

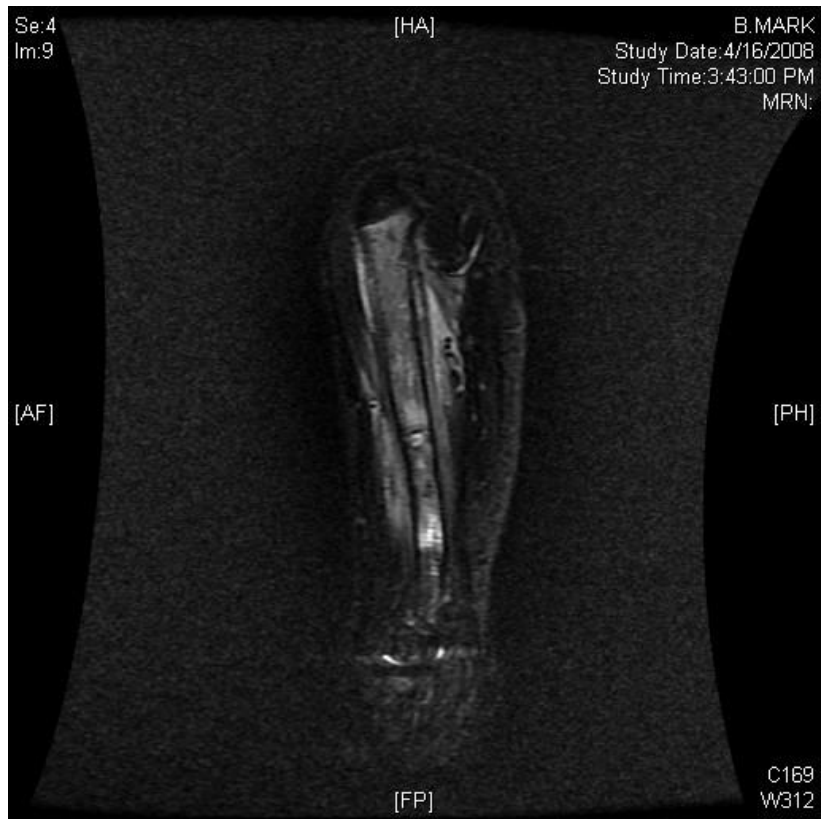

Figure 1: MRI image showing abnormal bone marrow signal within the majority of the humeral shaft with irregular cortical borders, periosteal reaction, and mottling of the diaphysis with contrast administration.

(a)

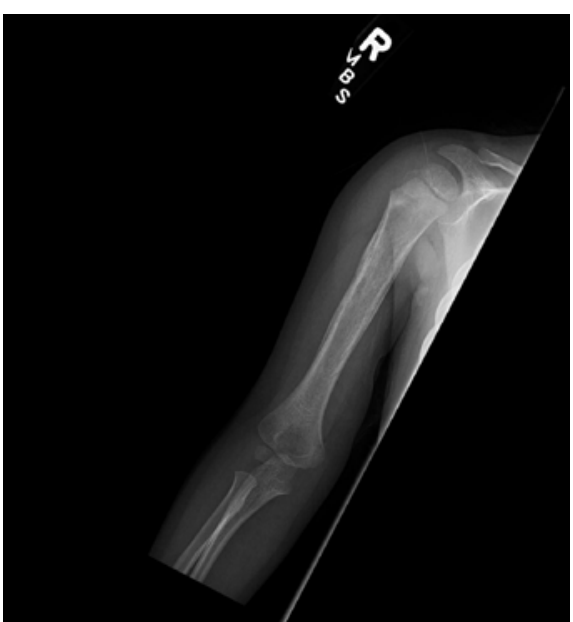

(b)

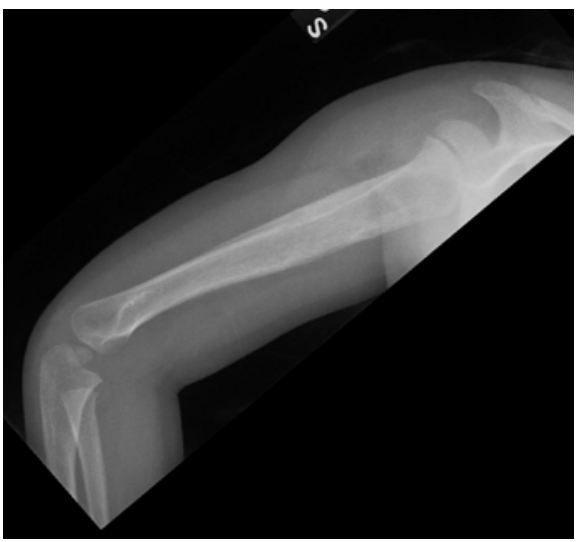

Figure 2: Anteroposterior and lateral radiographs of the humerus showing cortical remodeling of the humeral diaphysis with minimal heterogeneity of the medullary space. (a)

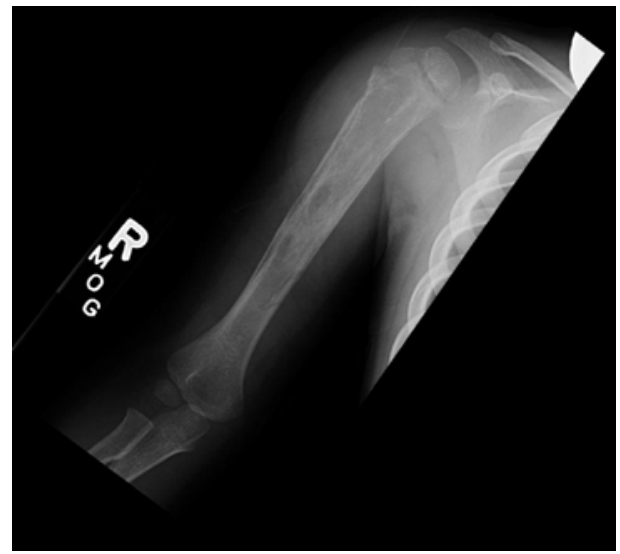

(b)

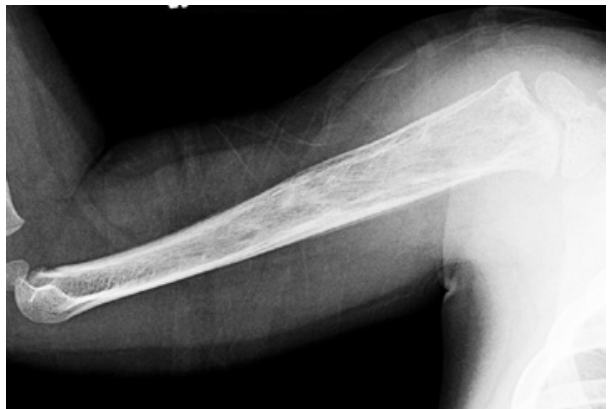

Figure 3: Anteroposterior and lateral radiographs of the humerus showing heterogeneous mineralization of the right humerus, progression of periosteal reaction, with development of new ill-defined lucencies in the proximal humerus.

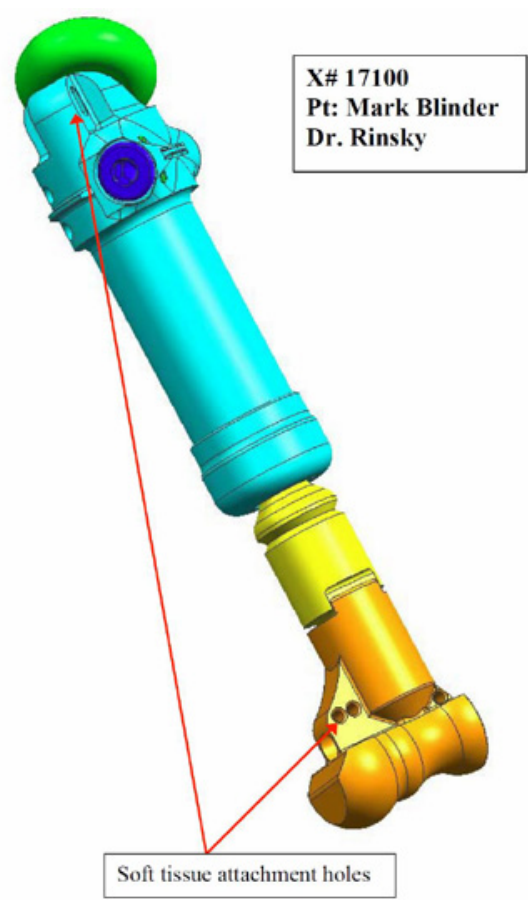

Figure 4a: Design schematic for the custom built prosthesis specifically designed according to the imaging measurements of the patient's humerus. The prosthesis was engineered to fit into the patient's upper arm and expand with the child's growth. 
The patient initiated multi-agent induction chemotherapy and imaging studies documented partial resolution of the lesion. Radical enbloc resection of the entire right humerus was planned due to the distal extent of tumor involvement. Since the patient was just three years of age, a standard prosthesis did not exist that could accommodate for the patient's specific anatomical parameters. A custom built prosthesis was specifically designed according to the imaging measurements of the patient's own humerus (Figure $4 \mathrm{a}-\mathrm{c}$ ). This humeral prosthesis was engineered to not only fit into the patient's upper arm but also to expand with the child's growth. In December of 2008, radical en-bloc resection of the entire right humerus was performed. The resection included the anterior $1 / 2$ of the deltoid, upper portion of the brachialis, and the medial head of the triceps. Extreme care was utilized in the surgical dissection to completely avoid violating the tumor and biopsy site using tumor surgery principles. Extraperiosteal dissection was utilized for the resection, taking care to protect and preserve the radial and ulnar nerves. Once the entire humerus was resected successfully, the custom built modular prosthesis was assembled and inserted into the empty space of the upper arm. (Figure 5a-d) Proximally, the prosthesis articulated well with the glenoid and with expansion of the prosthesis, the distal end articulated well with the proximal ulna.

The remaining rotator cuff tendons, teres major, latissimusdorsi, and deltoid attachments were re-established by repairing them onto a Dacron sleeve attached to the proximal portion of the prosthesis. The entire implanted prosthesis proved to be stable upon closure. Pathology examination demonstrated no residual viable tumor within the resected humerus, with clear margins. Postoperatively, the patient continued the multi-agent chemotherapy, which he completed 6 months later with no evidence of metastatic disease or recurrence. His wound healed uneventfully without any evidence of infection. Follow-up radiographs demonstrated a well-reduced humeral prosthesis both proximally and distally (Figure 6a,b). Expansion of the prosthesis of one centimeter was carried out one year after the completion of chemotherapy (18 months after resection).. At his most recent orthopaedic followup, two and one half years post op, the patient was doing well and was improving significantly with his function and motion. At his shoulder, the patient had approximately 40 degrees of active abduction, but passive total abduction up to 120 degrees. A second lengthening of one centimeter is planned. He lacked 10 degrees from full extension and flexed to 125 degrees at the elbow without pain. The patient has excellent hand function without any neurologic deficit continues to grow.

\section{Discussion}

Ewing's sarcoma in this age group is rare, however should be considered in children presenting with the constellation of signs and symptoms seen in our patient. As in our case and other reports in the literature, Ewing's sarcoma can masquerade as osteomyelitis. Multiple accounts of presumed, clinically diagnosed osteomyelitis have been documented that ultimately proved to be Ewing's sarcoma [8-12]. Several cases report transient resolution of symptoms after treatment with antibiotics, similar to our case [8-10]. This highlights the need for expedient surgical biopsy with path histological and cytogenetic examination of these lesions. Delay in definitive diagnosis and appropriate treatment may adversely affect clinical outcome. Ewing's sarcoma has retained the most unfavorable prognosis of all primary musculoskeletal tumors [17]. Prognosis of Ewing's sarcoma is highly dependent on clinically evident metastatic disease, which may be preventable with early detection and treatment with aggressive local disease control and systemic multidrug chemotherapy [2]. To our knowledge, this is the youngest patient in the literature to undergo total humeral resection and modular expandable prosthetic replacement for Ewing's sarcoma. In the past, amputation was the treatment of choice for these very young patients since limb salvage was previously considered a nonviable option [13]. This is due to their growth potential and difficulty in actively cooperating and participating in postoperative rehabilitation at such a young age. Since the proximal humerusphysis contributes to approximately $80 \%$ of the total growth of the humerus, resection of the proximalphysis prevents that growth, effectively leading to a limb length inequality [7] In this case, since the entire humerus ( both proximal and distal physes) had to be resected, there would be no possibility for any ongoinghumeral

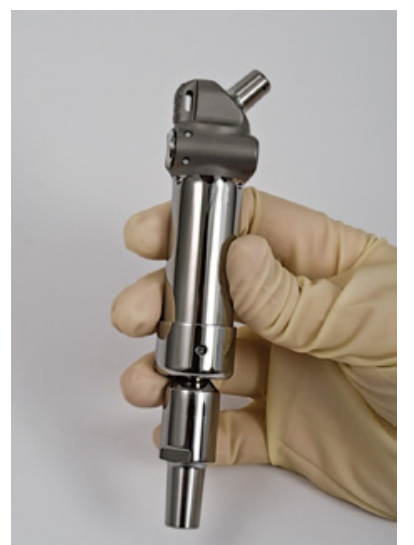

Figure 4b: Photograph of the modular humeral prosthesis, proximal humeral component. Top shows morse taper for prosthetic humeral head component. Bottom shows morse taper for distal humeral component.

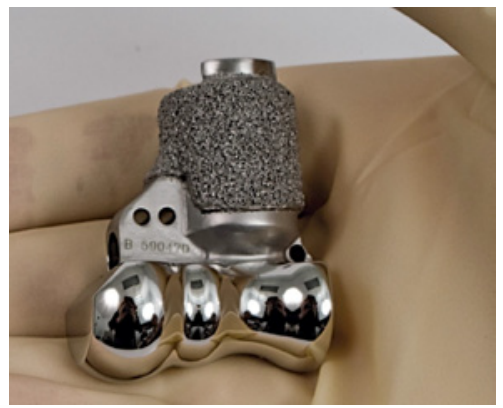

Figure 4c: Photograph of the modular humeral prosthesis, distal humeral component.

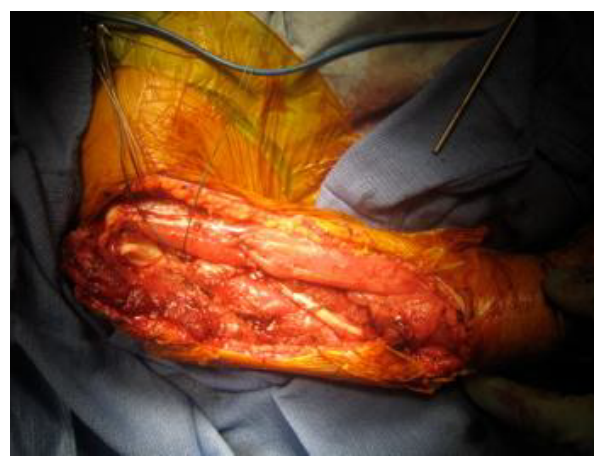

Figure 5a: Intraoperative photograph showing the empty space after the humerus was excised. Left shows the glenoid and right shows the elbow joint. The rotator cuff tendons and radial nerve are tagged. 
Citation: Park DY, Batista E, Rinsky LA (2011) Ewing's Sarcoma of the Humerus in a Three year-old boy Requiring Radical En-Bloc resection and Total Humeral Expandable Prosthetic Replacement: A Case Report. Surgery 1:101. doi:10.4172/2161-1076.1000101

Page 4 of 5

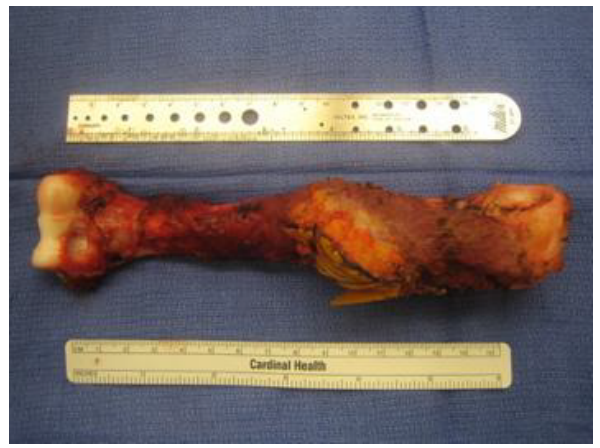

Figure 5b: Intraoperative photograph showing the entire humerus after excision.

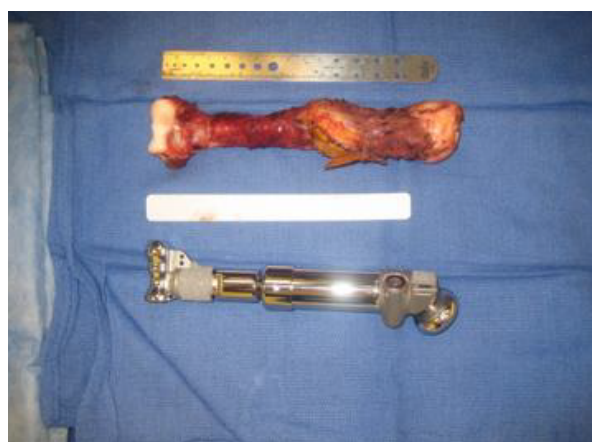

Figure 5c: Intraoperative photograph showing the excised humerus compared with the humeral prosthesis.

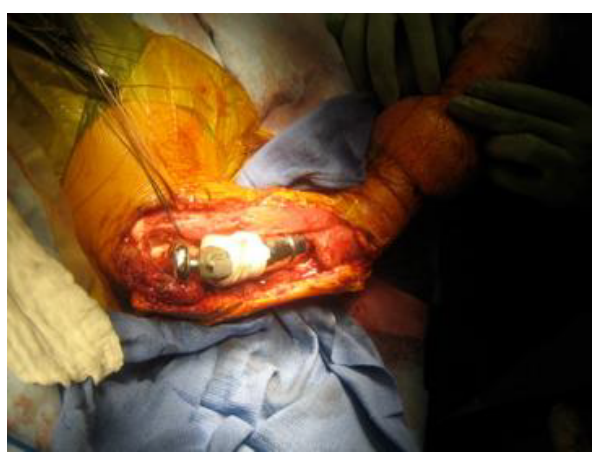

Figure 5d: Intraoperative photograph showing the humerus prosthesis seated in place within the patient's arm prior to closure.

growth. In the upper extremity however, maintenance of fine motor function is paramount in importance as compared to limb length, making inequality more of an issue cosmetically [18]. Tumor excision may include the rotator cuff and/or deltoid muscle, resulting in poor function of the salvaged shoulder. The challenge of limb salvage in very young children includes maintaining both some limb length and function after resection. The ultimate surgical goal is complete excision of the tumor with clear margins to reduce the possibility of recurrence and effect a cure.Limb salvage techniques such as allograft-prosthesis composite reconstruction have been utilized in proximal humerus lesions to reconstruct the rotator cuff and deltoid attachments essential for shoulder function [14]. However, this technique also would not address the eventual limb length inequality that would occur after resecting the proximal humerusphysis. In addition, tumor involvement in our case extended into the olecranon fossa and distal fixation would be tenuous and likely fail given the paucity of bone in this region.

Recently, modular expandable prostheses have been implanted for malignant bone tumors with satisfactory results $[7,13]$. These prosthetic designs required formal surgical procedures for expansion and may potentially introduce risks of infection, loosening, and catastrophic failure. In a study examining extensible endoprosthetic replacement of the humerus, 18 patients, aged between 6-12 years, underwent custom-made prosthetic replacement for osteosarcoma or Ewing's sarcoma with a median follow-up of 70 months [7]. No deep infections were encountered and only three superficial infections from

(a)

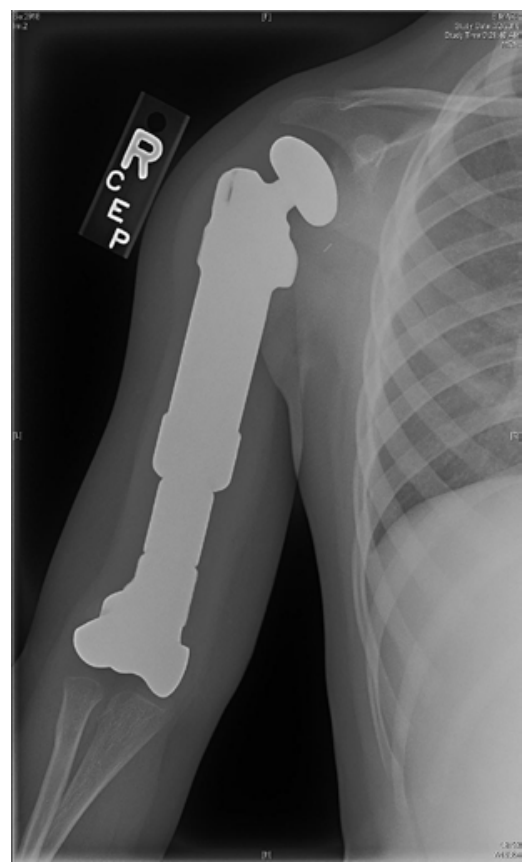

(b)

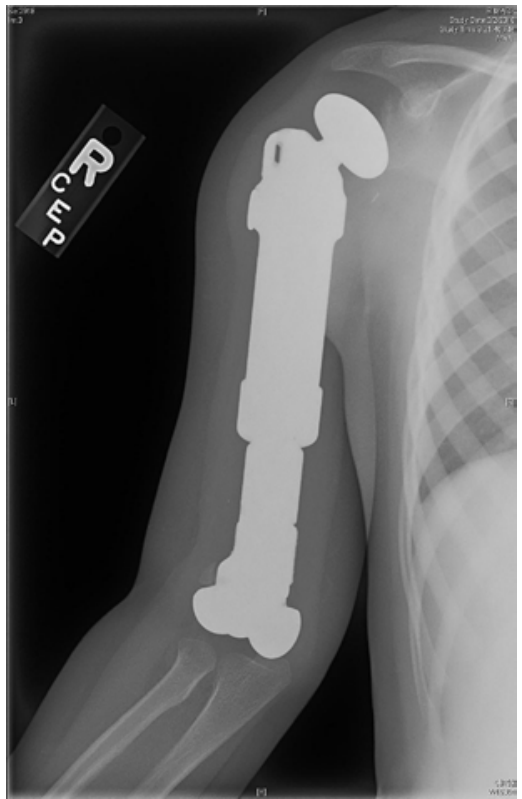

Figure 6: Postoperative anteroposterior and oblique radiographs showing the humeral prosthesis articulating with the glenoid proximally and the elbow joint distally. 
Citation: Park DY, Batista E, Rinsky LA (2011) Ewing's Sarcoma of the Humerus in a Three year-old boy Requiring Radical En-Bloc resection and Total Humeral Expandable Prosthetic Replacement: A Case Report. Surgery 1:101. doi:10.4172/2161-1076.1000101

subsequent extension procedures. There were three radial nerve palsies from the index resection procedure and none following the extension procedures. Mean total extension was $29.9 \mathrm{~mm}$ with 2 lengthening per year per patient. Estimated length discrepancy was $10 \mathrm{~mm}$ in most cases but up to $50 \mathrm{~mm}$ in 2 cases. However, these 2 cases refused further Lengthening since they were satisfied with their function. Functional results were satisfactory with a mean functional rating of $79.3 \%$ according to the Enneking system.

Current advancements in modular expandable prosthetic design allow non-invasive methods of lengthening the reconstructed limb. An innovative non-invasive expandable prosthetic design utilizes compressed spring energy that is slowly released when placed in an external electromagnetic field for expansion of the prosthesis $[15,16]$. A study of 15 patients with osteosarcoma of the knee demonstrated a mean MTS functional score of $90 \%$ at a mean follow-up of 21.5 months [16]. Patients averaged 4.3 lengthening procedures with $8.5 \mathrm{~mm}$ of length obtained with each procedure. Early experience of this design is promising and may avoid further surgical risk associated with invasive methods of expansion. We described a case of a three-year-old boy who initially was diagnosed and treated for osteomyelitis that ultimately proved to be Ewing's sarcoma of the right humerus. Differentiating between osteomyelitis and Ewing' sarcoma can be difficult and surgical biopsy with pathohistological and cytogenetic examination may be necessary for definitive diagnosis. Surgical reconstruction of very young children is challenging and expandable modular prosthetic replacement can provide a functional limb-salvage solution that minimizes limb length inequality after resection.

\section{References}

1. Heare T, Hensley MA, Dell'Orfano S (2009) Bone tumors: osteosarcoma and Ewing's Sarcoma. Curr Opin Pediatr 21: 365-372.

2. Maheshwari AV, Cheng EY (2010) Ewing sarcoma family of tumors. J Am Acad Orthop Surg 18: 94-107.

3. Arndt CA, Crist WM (1999) Common musculoskeletal tumors of childhood and Adolescence. N Engl J Med 341: 342-352.
4. Young JL Jr, Miller RW (1975) Incidence of malignant tumors in U. S. children. J Pediatr 86: 254-258.

5. Esiashvili N, Goodman M, Marcus RB Jr (2008) Changes in incidence and survival of Ewing sarcoma patients over the past 3 decades: Surveillance Epidemiology and End Results data. J Pediatr Hematol Oncol 30: 425-430.

6. Maygarden SJ, Askin FB, Siegal GP, Gilula LA, Schoppe J, et al. (1993) Ewing sarcoma of bone in infants and toddlers. A clinic pathologic report from the Intergroup Ewing's Study. Cancer 71: 2109-2118.

7. Ayoub KS, Fiorenza F, Grimer RJ, Tillman RM, Carter SR (1999) Extensible endoprostheses of the humerus after resection of bone tumours. J Bone Joint Surg $\mathrm{Br}$ 81: 495-500.

8. Durbin M, Randall RL, James M, Sudilovsky D, Zoger S (1998) Ewing's sarcoma Masquerading as osteomyelitis. Clin OrthopRelat Res 357: 176-185.

9. Nance CL Jr, Roberts WM, Miller GR (1967) Ewing's sarcoma mimicking Osteomyelitis. South Med J Oct 60: 1044-1050.

10. Tow BP, Tan MH (2005) Delayed diagnosis of Ewing's sarcoma of the right humerus Initially treated as chronic osteomyelitis: a case report. J OrthopSurg 13: $88-92$

11. Lyall HA, Constant CR, Wraight EP (1993) Case report: Ewing's sarcoma in distal tibial metaphysis mimicking osteomyelitis. ClinRadiol. 48: 140-142.

12. Rasit AH, Sharaf I, Rahman HA (2001) Ewing's sarcoma of the talus in a fouryear-old child. Med J Malaysia 56: 86-88.

13. Neel MD, Letson GD (2001) Modular endoprostheses for children with malignant bone Tumors. Cancer Control 8: 344-348.

14. Abdeen A, Hoang BH, Athanasian EA, Morris CD, Boland PJ, et al. (2009) Allograft-prosthesis composite reconstruction of the proximal part of the Humerus: functional outcome and survivorship. J Bone Joint Surg Am 91 2406-2415.

15. Wilkins RM, Soubeiran A (2001) The Phoenix expandable prosthesis: early American Experience. Clin Orthop Relat Res 382: 51-58.

16. Neel MD, Wilkins RM, Rao BN, Kelly CM (2003) Early multicenter experience with a Noninvasive expandable prosthesis. Clin Orthop Relat Res 415: 72-81.

17. Fouda, A, Mansour, A, Al-Tonbary, Y. The many faces of Ewing sarcoma: difficult to diagnose pediatric cases. Hematology Oncology Stem Cell Therapy 2: 411-417.

18. Yaw KM (1999) Pediatric Bone Tumors. Seminars in Surgical Oncology 19 173-183. 